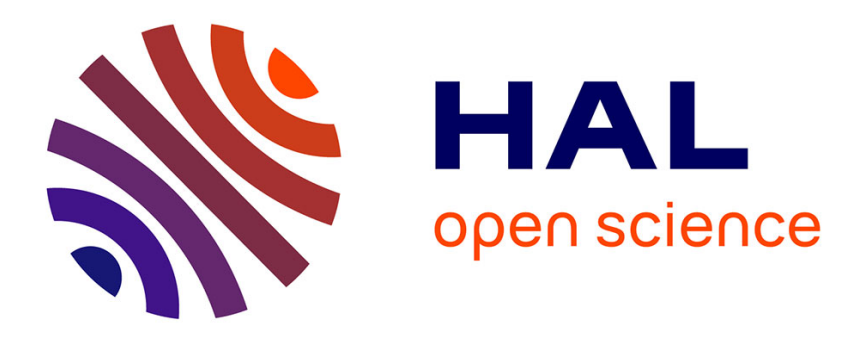

\title{
Thermodynamic and kinetic analysis of non elastic deformation in polymeric glasses
}

\author{
B. Escaig, J.M. Lefebvre
}

\section{To cite this version:}

B. Escaig, J.M. Lefebvre. Thermodynamic and kinetic analysis of non elastic deformation in polymeric glasses. Revue de Physique Appliquée, 1978, 13 (6), pp.285-292. 10.1051/rphysap:01978001306028500 . jpa-00244451

\section{HAL Id: jpa-00244451 https://hal.science/jpa-00244451}

Submitted on 1 Jan 1978

HAL is a multi-disciplinary open access archive for the deposit and dissemination of scientific research documents, whether they are published or not. The documents may come from teaching and research institutions in France or abroad, or from public or private research centers.
L'archive ouverte pluridisciplinaire HAL, est destinée au dépôt et à la diffusion de documents scientifiques de niveau recherche, publiés ou non, émanant des établissements d'enseignement et de recherche français ou étrangers, des laboratoires publics ou privés. 


\title{
REVUE DE PHYSIQUE APPLIQUÉE
}

Classification

Physics Abstracts

$81.20 \mathrm{~S}-81.40 \mathrm{~L}$

\section{THERMODYNAMIC AND KINETIC ANALYSIS OF NON ELASTIC DEFORMATION IN POLYMERIC GLASSES}

\author{
B. ESCAIG and J. M. LEFEBVRE \\ Laboratoire de Structures et Propriétés de l'Etat Solide $\left({ }^{*}\right)$ \\ Université des Sciences et Techniques de Lille, B. P. 36, 59650 Villeneuve d'Ascq, France
}

(Reçu le 21 décembre 1977, révisé le 3 mars 1978, accepté le 13 mars 1978)

\begin{abstract}
Résumé. - L'article étend aux polymères solides l'approche thermodynamique et cinétique utilisée en plasticité cristalline avec essentiellement en vue l'application à la limite élastique des polymères vitreux due en large part au dépliement des chaînons le long de grossières bandes de cisaillement. On définit successivement les paramètres d'activation à l'équilibre thermodynamique, puis la cinétique d'activation et les diverses grandeurs opérationnelles et procédures expérimentales. On montre que les contributions propres à l'orientation des chaînons induite sous contraintes sont en général négligeables.

Abstract. - This paper is aimed at extending to polymeric solids the thermodynamics and kinetics of activated plasticity as it has been used in crystals for about ten years. Application to thermally activated yielding in glassy polymers, due to the uncoiling of chain bonds within crude shear bands, producing an anelastic (pseudo-plastic) strain, is particularly stressed. Definitions of the activation parameters are given based on equilibrium quasi-static thermodynamics, and contributions to the total activation entropy and free energy from the strain induced bond orientation are shown to be generally negligible. The kinetics of barrier overcoming are considered and the operational quantities and experimental procedures are discussed.
\end{abstract}

\section{List of main notations}

$\Delta G_{\mathrm{a}}$ Activation free energy

$\Delta S_{\mathrm{a}}$ Activation entropy

$\Delta H_{\mathrm{a}}$ Activation enthalpy

$\sigma_{\mathrm{a}} \quad$ Applied stress

$V_{\mathrm{a}}$ Activation volume

$V_{\mathrm{c}}$ Critical nucleus volume

$V_{0} \quad$ Fully expanded nucleus volume

$v_{\mathrm{e}} \quad$ Number of active chains

$\mu \quad$ Shear Modulus

$\Delta e_{0}$ Elementary strain in the nucleus

$\varepsilon_{\mathrm{s}} \quad$ Entropic strain

$S \quad$ Reduced stress $=\sigma_{\mathrm{a}} \times\left(\mu_{0} / \mu\right)$

$v_{\mathrm{D}} \quad$ Bond vibration frequency

$v_{\mathrm{N}}$ Correlated vibrations frequency

1. Introduction. - Amorphous polymers can experience a durable deformation in the glassy phase, that is at temperatures below the glass transition [1]. Up to a certain stress, called the yield stress, the strain is

(*) Laboratoire associé au C. N. R. S. mainly elastic in the sense that only Hooke internal energy changes are involved and goes down to zero after unloading. Beyond yielding, a remanent strain still persists at zero load for some time, which recovers at an observable rate if enough time or temperature is allowed. This anelastic strain is clearly of an entropic nature ; it is merely due to the uncoiling of chain bonds within crude shear bands with a strong increase in bond orientation, as demonstrated by birefringence measurements [2], i. e. to a major decrease in entropy. It has its exact parallel in crystalline deformation, although to a much smaller extent, with the anelastic strain produced by stress ordering of interstitial impurities in a b. c. c. lattice, like carbon in iron for example. If the recovery time is very long, which is currently the case for thermoplastics, this anelastic behaviour may resemble an apparent plasticity. Occasionally a true, unrecoverable plasticity can also develop in special conditions, e. g. injection moulding, corresponding to a flow of the center of gravity of polymer molecules without any change in internal energy nor in entropy so that a true permanent strain is retained at zero load. 
Yielding is a strongly thermally activated process in glassy polymers $[1,3,4]$. It is thus of great interest to know how the strain rate is related to stress and temperature, and firstly whether a simple Arrhenius law is obeyed, with the meaning that only one mechanism prevails on a molecular scale. The experimental determination of the stress and temperature dependence of the strain rate is then aimed at an assessment of the characteristic features for this mechanism : energy and spatial extension. However, such an analysis needs to be conducted carefully, with a precise definition of concepts, activation parameters and assumptions, and from a consistent set of data. For example, most of the published studies $[3,4,5]$ derive energies which are actually activation enthalpies, and are not the relevant mechanism energies ; the latter are Gibbs free energies, hence are only obtainable if an evaluation of the main activation entropy contributions are made ; in the case of polymers, this leads to appreciable corrections $[6,7]$ owing to the strong dependence of the elastic constants on temperature in the range usually investigated. The same can be said about the measured activation volume, the meaning of which needs a precise thermodynamic identification.

It is the aim of this paper to extend to polymeric solids, the thermodynamics and kinetics of activated plasticity as it is used in crystals [8-11]. The general framework is given in part 2 , with the specific assumptions required for polymeric materials. It is shown in part 3 that the activation parameters (energy, entropy, volume) can be deduced from equilibrium quasistatic thermodynamics which impose inter-relations between them $[9,12]$. Also, contributions to the total activation entropy and free energy from the straininduced bond orientation (through rubberlike terms) are shown to be generally negligible as compared with the Hooke elastic terms. The kinetics of barrier overcoming are considered in part 4 , while operational quantities and procedures are dealt with in part 5 , with a special interest in the self-consistency of experimental data, i. e. the check of validity of a simple Arrhenius law featuring a single prevailing mechanism. In this regard, it is emphasized that no experimental support to a given model can be claimed from this kind of measurement, if the consistency of all the measured activation parameters has not been first checked by a complete analysis. Some of the results we obtained recently in our laboratory during the course of an experimental investigation on polystyrene and polymethylmethacrylate [7] are given to illustrate this point. A more complete account of these results will be published soon elsewhere.

2. General assumptions for the thermally activated yield and flow in polymers. - We do not intend to propose here a specific mechanism of deformation, but rather some plausible features which frame a general approach.

In an annealed glassy polymer, any molecular seg- ment is in some kind of energy well, or cage, formed by its nearest neighbours, the curvature of which determines the high value of the elastic constants measured in the glassy phase. It is of course, recognized that the glass structure is not a true equilibrium structure because of the slow decrease of its excess free volume. However, the characteristic relaxation time $\tau_{R}$ of this slow ageing is relatively large, depending on the annealing treatment ; it should be at least of order $10-10^{2}$ seconds, for a free volume equal to the glass transition value $\left({ }^{1}\right)$ and probably larger as the excess volume is ageing down at temperatures below that point. Therefore, the cage structure of the glass can be considered as being in a quasi equilibrium state for any polymer response that does not require a time $\tau$ much larger than $\tau_{R}$; this is the case for the thermal activation of plasticity, as is shown in part $4\left(\tau_{\text {def }} \simeq 10^{-3} \mathrm{~s}\right)$.

At very low temperatures, yield stresses of the order $0.1 \mu$ ( $\mu$, the shear modulus) are reported for various thermoplastics $[13,14]$ suggesting that yielding might occur at a stress equal to the Frenkel theoretical shear strength [14]. This stress is such that chain bonds can slip past each other at any place within the shear band. At higher temperatures and lower stresses, a similar bond shear can take place locally, in a small region of volume $V$ in the polymer, with the aid of thermal fluctuations ; under the applied stresses, yielding starts spreading further from those nuclei which have grown past a critical, unstable size. The height of the consequent energy barrier to expansion can be deduced from the critical configuration and the elastic reaction it develops from the unsheared surrounding matrix, as several specific models have proposed $[13,14,15]$. It is enough for our analysis to recognize that the shear uncoiling of chain bonds through the cage potential wells starts from some critical nuclei of volume $V_{c}$, within which correlated motions of segments occur when they form and small enough to be efficiently activated by thermal fluctuations.

Changes in nucleus energy during their stress-aided, thermally activated growth can be described by standard quasi-static thermodynamics, while the overcoming of these energy barriers can be analysed by the reaction rate theory. One has to define a reaction coordinate; for the sake of simplicity and because bonds uncoiling is the basic cause of anelastic behaviour, it is appropriate to take here the entropic strain $\varepsilon_{\mathrm{s}}$ (which is a state variable) produced by uncoiling

(1) Consider the time $\tau_{\mathrm{R}}$ needed for a neighbour to leave the cage, thus perturbing strongly its energy well. One can estimate :

$$
\tau_{\mathrm{R}} \simeq(p \varphi)^{-1} \exp \left(\alpha T_{\mathrm{g}} / T\right)
$$

where $p$ is the number of neighbours forming the cage, $p \simeq 10$, $\varphi$ is the free volume dependent jump frequency of a molecular segment, $\varphi=0.1 \mathrm{~s}^{-1}$ for equilibrium at the glass temperature $T_{\mathrm{g}}$ [16] and $\alpha k T_{\mathrm{g}}=U_{\mathrm{m}}$, being a motion energy in the glassy phase for a segment to jump into an adjacent hole, $\alpha \simeq 2$ or 3 . These values lead to a minimum estimate for $\tau_{\mathrm{R}} \simeq 10-100 \mathrm{~s}$, with $\left(T_{\mathrm{g}} / T\right) \gtrsim(400 / 300)$. 
chain bonds within the shear nucleus (other choices could also be made, as for example choosing the nucleus size itself $V$, which is proportionnal to $\varepsilon_{\mathrm{s}}$ ). Because the thermodynamical system is taken as the whole polymer sample, the strain to be considered here is the sample average strain $\varepsilon_{\mathrm{s}}$ corresponding to an entropic strain increment $\Delta e_{0}$ in the nucleus volume $V$ :

$$
\varepsilon_{\mathrm{s}}=\frac{V}{V_{\mathrm{TOT}}} \Delta e_{0}
$$

where $V_{\text {TOт }}$ is the total volume of sample. Most plausibly, $\Delta e_{0}$ has to be considered as a given molecular cage parameter, of the order unity [14] and the variable during reaction is $V$ :

$$
\mathrm{d} \varepsilon_{\mathrm{s}}=\left(\Delta e_{0} / V_{\text {TOT }}\right) \mathrm{d} V .
$$

3. Thermodynamic definitions of the activation parameters. - The aim of this part is to give proper definitions to the activation parameters and to show how these are related to the two directly available by experiment, i. e. the activation volume and enthalpy (see part 5). We merely transpose in the following the fundamentals of thermodynamical analysis formulated by Schoeck for crystals [9].

Let us consider first the increase in free energy of a polymer sample during a reversible and isothermal variation $\mathrm{d} \varepsilon_{\mathrm{s}}$. The internal energy change is :

$$
\mathrm{d} U=T \mathrm{~d} S+\delta W
$$

$\mathrm{d} S$ is the change in entropy of the polymer from changes in total volume, vibrational spectrum and segment orientation. $\delta W$ is the reversible work done by the applied stresses on the polymer, due to the energy barrier opposing the nucleus expansion. Apart from the pressure term $-P \mathrm{~d} V_{\text {TOT }}$, it is formed by a work term $\delta W_{\mathrm{H}}$, originated by the increase in the Hooke elastic energy stored in the total volume and a rubber-like (entropic) work term $\delta W_{\mathrm{s}}$, originated by the deviation to maximum entropy from the additional uncoiling of chain bonds in the growing nucleus. $\delta W_{\mathrm{H}}$ depends merely on nucleus size and temperature (through the elastic constants) and is assumed not to depend explicity on the applied stress :

$$
\delta W_{\mathrm{H}}=h\left(\varepsilon_{\mathrm{s}}, T\right) \mathrm{d} \varepsilon_{\mathrm{s}}\left({ }^{2}\right)
$$

$\delta W_{\mathrm{s}}$ can be written :

$$
\delta W_{\mathrm{S}}=-T \mathrm{~d} S_{\mathrm{e} 1}
$$

with $\mathrm{d} S_{\mathrm{el}}$, the appropriate entropy drop caused by extending the shear band $\Delta e_{0}$ (for simple shear) by a volume $\mathrm{d} V[18]$ :

$$
\mathrm{d} S_{\mathrm{el}}=-\frac{k v_{\mathrm{e}} \Delta e_{0}^{2}}{2} \mathrm{~d} V=-V_{\text {Tот }}\left(\frac{k v_{\mathrm{e}} \Delta e_{0}}{2}\right) \mathrm{d} \varepsilon_{\mathrm{s}}
$$

(2) Eq. (2) should be applied to the only Hooke strain $\mathrm{d} \varepsilon_{\mathrm{H}} \neq \mathrm{d} \varepsilon_{\mathrm{s}}$. However, one can very generally assume proportionality between $\mathrm{d} \varepsilon_{\mathrm{H}}$ and $\mathrm{d} V$, and so, from eq. (1), between $\mathrm{d} \varepsilon_{\mathrm{H}}$ and $\mathrm{d} \varepsilon_{\mathrm{s}}$. $v_{\mathrm{e}}$ being the number of active chains (entanglements) in unit volume; so that :

$$
\mathrm{d} U=T \mathrm{~d} S-P \mathrm{~d} V_{\text {TOT }}+\delta W_{\mathrm{H}}+\delta W_{\mathrm{S}} .
$$

Hence the change in the Gibbs free energy of the polymer sample, $G_{0}=U-T S+P V_{\text {TOT }}$, under the conditions $P=$ const., $T=$ const., can be written as :

$\mathrm{d} G_{0}=\mathrm{d} U-T \mathrm{~d} S+P \mathrm{~d} V_{\text {TOT }}=\delta W_{\mathrm{H}}+\delta W_{\mathrm{S}}$.

Now, under the conditions $P=$ const., $T=$ const., and $\sigma_{\mathrm{a}}=$ const., $\sigma_{\mathrm{a}}$ being the external applied stress, the evolution is controlled by the thermodynamic potential $G=G_{0}-W^{\prime}\left(\sigma_{\mathrm{a}}, \varepsilon_{\mathrm{s}}\right)$ where $W^{\prime}$ is the work done by the constant stress $\sigma_{\mathrm{a}}$ during deformation $\varepsilon_{\mathrm{s}}$, that is the Gibbs free energy of the closed system formed by the polymer sample plus the external sources of stress. During the variation $\mathrm{d} \varepsilon_{\mathrm{s}}, \delta W^{\prime}=\sigma_{\mathrm{a}} V_{\text {TOT }} \mathrm{d} \varepsilon$; the strain $\mathrm{d} \varepsilon$ is made of $\mathrm{d} \varepsilon_{\mathrm{s}}$, plus an elastic Hooke term related to bond distortions, plus eventually a true plastic flow term. Assuming these two latter to be negligible as compared with $\mathrm{d} \varepsilon_{\mathrm{s}}, \mathrm{d} \varepsilon \simeq \mathrm{d} \varepsilon_{\mathrm{s}}$ :

$$
\begin{aligned}
\mathrm{d} G & =\delta W_{\mathrm{H}}+\delta W_{\mathrm{S}}-\sigma_{\mathrm{a}} V_{\text {TOT }} \mathrm{d} \varepsilon_{\mathrm{s}} \\
& =\left[\left(\frac{\partial G_{0}}{\partial \varepsilon_{\mathrm{s}}}\right)_{T, P}-\sigma_{\mathrm{a}} V_{\text {TOT }}\right] \mathrm{d} \varepsilon_{\mathrm{s}}
\end{aligned}
$$

Finally the activation free energy of reaction is given by the integral over the barrier :

$$
\Delta G_{\mathrm{a}}=\int_{\varepsilon_{\mathrm{s}}(0)}^{\varepsilon_{\mathrm{s}}(c)}\left[\left(\frac{\partial G_{0}}{\partial \varepsilon_{\mathrm{s}}}\right)_{T, P}-\sigma_{\mathrm{a}} V_{\mathrm{TOT}}\right] \mathrm{d} \varepsilon_{\mathrm{s}}
$$

$\varepsilon_{\mathrm{s}}(0)$ and $\varepsilon_{\mathrm{s}}(c)$ define respectively the initial equilibrium state of the polymer under stress and the saddle point configuration at the top of the barrier; they are thus the roots of the equation :

$$
\left(\frac{\partial G_{0}}{\partial \varepsilon_{\mathrm{s}}}\right)_{T, P}=\sigma_{\mathrm{a}} V_{\mathrm{TOT}}, \text { for } \varepsilon_{\mathrm{s}}=\varepsilon_{\mathrm{s}}(0) \text { or } \varepsilon_{\mathrm{s}}(c) \text {. }
$$

Definitions of the activation entropy $\Delta S_{\mathrm{a}}$ and activation volume $V_{\mathrm{a}}$ follow from the Second Law of Thermodynamics :

$$
\begin{gathered}
\mathrm{d}\left(\Delta G_{\mathrm{a}}\right)_{P}=-\Delta S_{\mathrm{a}} \mathrm{d} T-V_{\mathrm{a}} \mathrm{d} \sigma_{\mathrm{a}} \\
\Delta S_{\mathrm{a}}=-\left(\frac{\partial \Delta G_{\mathrm{a}}}{\partial T}\right)_{P, \sigma_{\mathrm{a}}} ; \quad V_{\mathrm{a}}=-\left(\frac{\partial \Delta G_{\mathrm{a}}}{\partial \sigma_{\mathrm{a}}}\right)_{T, P} .
\end{gathered}
$$

More explicit expressions can be obtained if equations (2), (3), (5), (6) are inserted and the derivations performed. Note that the bounds $\varepsilon_{\mathrm{s}}(0)$ and $\varepsilon_{\mathrm{s}}(c)$ in equation (6) are functions of $\sigma_{\mathrm{a}}$ and $T$ (through the elastic constants) as shown in equation (7). However, as Schoeck first pointed out [9], their contributions to the derivatives vanish according to equation (7), for example :

$$
\frac{\partial \varepsilon_{\mathrm{s}}(c)}{\partial T^{\prime}} \cdot\left[\frac{\partial G_{0}}{\partial \varepsilon_{\mathrm{s}}}-\sigma_{\mathrm{a}} V_{\text {TOT }}\right]_{\varepsilon_{\mathrm{s}}=\varepsilon_{\mathrm{s}}(c)}=0 .
$$


Therefore, one has only to derive equation (6) under the integral $\left({ }^{3}\right)$ :

$$
\begin{aligned}
\Delta S_{\mathrm{a}} & =-\int_{0}^{c} \frac{\partial}{\partial T}\left(\frac{\partial G_{0}}{\partial \varepsilon_{\mathrm{s}}}\right)_{\sigma_{\mathrm{k}}} \mathrm{d} \varepsilon_{\mathrm{s}} ; \\
V_{\mathrm{a}} & =V_{\text {TOT }} \Delta \varepsilon_{\mathrm{s}}\left(\sigma_{\mathrm{a}}, T\right)=\Delta e_{0} V_{\mathrm{c}}\left(\sigma_{\mathrm{a}}, T\right)
\end{aligned}
$$

with $\Delta \varepsilon_{\mathrm{s}}=\varepsilon_{\mathrm{s}}(c)-\varepsilon_{\mathrm{s}}(0)\left({ }^{4}\right)$. This expression for $V_{\mathrm{a}}$ shows that it varies as $V_{\mathrm{c}}$, the nucleus volume at the saddle point, or, more physically, as the number of segments fluctuating in a correlated way for a successful event to occur. In activated dislocation slip theory, it would be proportional to the area swept out by the moving dislocation segment up to the top of the barrier. In both cases, this result relies on the assumption of a rigid barrier, the profile of which, $\mathrm{d} G_{0}=\delta W_{\mathrm{H}}+\delta W_{\mathrm{S}}$, does not depend explicitly on stress $\sigma_{\mathrm{a}}$. If the simple estimate $\Delta e_{0} \simeq 1$ is accepted, as plausible molecular models suggest [14], then measuring $V_{\mathrm{a}}$ allows us to reach an order of magnitude estimate of $V_{c}$, a characteristic parameter of the process.

The explicit expression of $\Delta S_{\mathrm{a}}$ requires a further assumption regarding the temperature dependence of $h\left(\varepsilon_{\mathrm{s}}, T\right)$ in equation (2). Since it represents a stored elastic energy, it is reasonable to take it proportional to the shear modulus $\mu(T)$ as its only temperature dependent term [6,9]. In the cases where this is justified :

$$
\frac{\partial}{\partial T}\left(\delta W_{\mathrm{s}}+\delta W_{\mathrm{H}}\right)_{\sigma_{\mathrm{a}}}=\frac{h}{\mu} \frac{\mathrm{d} \mu}{\mathrm{d} T} \mathrm{~d} \varepsilon_{\mathrm{s}}-\mathrm{d} S_{\mathrm{el}}
$$

so that, after some straightforward calculations it comes :

$$
\begin{aligned}
& \Delta S_{\mathrm{a}}=\Delta S_{\mu}+\Delta S_{\mathrm{el}} ; \\
& \Delta S_{\mu}=-\frac{1}{\mu} \frac{\mathrm{d} \mu}{\mathrm{d} T} \frac{\Delta H_{\mathrm{a}}+\sigma_{\mathrm{a}} V_{\mathrm{a}}}{1-\frac{T}{\mu} \frac{\mathrm{d} \mu}{\mathrm{d} T}} \\
& \Delta S_{\mathrm{el}}=-\frac{k\left(v_{\mathrm{e}} V_{\mathrm{c}}\right) \Delta e_{0}^{2}}{2}
\end{aligned}
$$

with $\Delta H_{\mathrm{a}}=\Delta G_{\mathrm{a}}+T \Delta S_{\mathrm{a}}$, the activation enthalpy.

The activation free energy can also be expressed in terms of $\Delta H_{\mathrm{a}}$ and $V_{\mathrm{a}}$, under the preceding assumptions,

(3) For the sake of simplicity the temperature dependence of $V_{\text {тот }}$ has been omitted from the expression of $\Delta S_{\mathrm{a}}$. It can be shown it is negligible compared to the one of the elastic constants [6] since $\left(\mathrm{d} \ln V_{\text {тот }} / \mathrm{d} T\right)$ is smaller than $(\mathrm{d} \ln \mu / \mathrm{d} T)$ by at least a factor of ten for thermoplastics.

(4) Note the equivalence with the general definition of $V_{a}$ given by $\mathrm{Li}[12,17]$ as the local additional shear strain $\left[\Delta \varepsilon_{\mathrm{ad}}\right]$ in a volume element produced during the activation process, integrated throughout the volume, i. e. : $V_{\mathrm{a}}=\int \Delta \varepsilon_{\mathrm{ad}} \mathrm{d} v$. Introducing the strain average $\Delta \varepsilon_{\mathrm{s}}=V_{\text {TOT }}^{-1} \int \Delta \varepsilon_{\text {ad }} \mathrm{d} v$, gives $V_{\mathrm{a}}=V_{\text {Tот }} \Delta \varepsilon_{\mathrm{s}}$. The use of $\Delta e_{0}$ replaces the continuous distribution $\Delta \varepsilon_{\mathrm{ad}}(x)$ by a square profile : $\Delta \varepsilon_{\mathrm{ad}}=0$ outside the critical nucleus, $\Delta \varepsilon_{\mathrm{ad}}=\Delta e_{0}$ within it, such that :

$$
\int_{\text {tot. vol. }} \Delta \varepsilon_{\mathrm{ad}} \mathrm{d} v=\int_{\text {crit. nucl. }} \Delta e_{0} \mathrm{~d} v=V_{\mathrm{c}} \Delta e_{0}=V_{\mathrm{a}} .
$$

since $\Delta G_{\mathrm{a}}=\Delta H_{\mathrm{a}}-T \Delta S_{\mathrm{a}}$. It follows from equation (10) :

$$
\begin{aligned}
\Delta G_{\mathrm{a}} & =\Delta G_{\mu}+\Delta G_{\mathrm{el}} \\
\Delta G_{\mu} & =\frac{\Delta H_{\mathrm{a}}+\left(\frac{T}{\mu} \frac{\mathrm{d} \mu}{\mathrm{d} T}\right) \sigma_{\mathrm{a}} V_{\mathrm{a}}}{1-\frac{T}{\mu} \frac{\mathrm{d} \mu}{\mathrm{d} T}} \\
\Delta G_{\mathrm{el}} & =-T \Delta S_{\mathrm{el}}=\frac{k T\left(v_{\mathrm{e}} V_{\mathrm{c}}\right) \Delta e_{0}^{2}}{2} .
\end{aligned}
$$

The barrier height itself, $\Delta G_{0}$, may be of some interest for characterizing the process :

$$
\Delta G_{0}=\int_{0}^{c}\left(\frac{\partial G_{0}}{\partial \varepsilon_{\mathrm{s}}}\right)_{T, P} \mathrm{~d} \varepsilon_{\mathrm{s}}=\Delta G_{\mathrm{a}}+\sigma_{\mathrm{a}} V_{\mathrm{a}} .
$$

Unfortunately, there is no general argument to keep $\Delta G_{0}$ constant as stress and temperature of the experiment vary, eventhough experimental data may incidentally yield a constant plot $[5,6,7]$ over a certain range; this makes any extrapolation to zero stress or temperature somewhat hypothetic. For example, it is easy to verify from the preceding equations :

$$
\begin{aligned}
& \left(\frac{\partial \Delta G_{0}}{\partial \sigma_{\mathrm{a}}}\right)_{T, P}=\sigma_{\mathrm{a}}\left(\frac{\partial V_{\mathrm{a}}}{\partial \sigma_{\mathrm{a}}}\right)_{T, P} ; \\
& \left(\frac{\partial \Delta G_{0}}{\partial T}\right)_{\sigma_{\mathrm{a}}, P}=-\Delta S_{\mathrm{a}}+\sigma_{\mathrm{a}}\left(\frac{\partial V_{\mathrm{a}}}{\partial T}\right)_{\sigma_{\mathrm{a}}, P} .
\end{aligned}
$$

Two last remarks deserve attention. First, the contributions from rubber-like terms, $\Delta S_{\mathrm{e} 1}$ or $\Delta G_{\mathrm{el}}$, are in general negligible as compared with crystal-like terms $\Delta S_{\mu}$ or $\Delta G_{\mu}$. This is because the former are proportional to the number of entanglements in the nucleus, $v_{\mathrm{e}} V_{\mathrm{c}}$. It is well known that the mechanical response of plastics in the glassy state depends generally on very local motions of the chain segments $[16 b]$; consistently, experimental values of $V_{\mathrm{a}}$, therefore also $V_{\mathrm{c}}$, represent at most about ten individual segments $[3,7]$; therefore $v_{\mathrm{e}} V_{\mathrm{c}} \simeq 2$ or 3 even though active entanglements are probably very close to one another in the glassy phase. This makes $\Delta S_{\mathrm{el}}$ of order $k$ and $\Delta G_{\mathrm{el}}$ of order $k T$. Since at all but the higher temperatures, $\Delta G_{\mathrm{a}}$ is found equal to about $20 k T$, it follows that $\Delta G_{\text {el }} \ll \Delta G_{\mu}$; on the contrary $\Delta S_{\mathrm{a}}$ increases generally with temperature much more than $\Delta S_{\mathrm{e} 1}$ does, so that $\Delta S_{\mathrm{e} 1}$ might have some influence at low temperatures.

The second point is to emphasize the contribution of the temperature dependence of elastic constants to activation entropy. Not only the thermal changes in the long wave length vibrational modes of the glass contribute, but also all the modulus relaxations, i. e. those secondary transitions $(\beta, \gamma, \ldots$ processes $)$ which accomodate strain by local chain backbone or side group motion, if time and temperature allow them to occur. In this regard, the $T$-dependence of the shear modulus to be considered there, is the one at a frequency $v \simeq \tau_{\mathrm{def}}^{-1}$ corresponding to the characteristic 
time of the activation process, that is $v \simeq 10^{3} \mathrm{~s}^{-1}$ according to part 4 .

4. Kinetics of the activation rate. - The macroscopic strain rate produced by the statistical development of critical shear nuclei can be estimated by simple kinetic arguments :

$$
\dot{\varepsilon}=N \varepsilon_{0} R=\rho \Delta e_{0} V_{0} R
$$

It contains the number of active sites where nuclei may grow, $N=\rho V_{\text {Tот }}$ in the total volume, the average strain achieved with each successful activation event, $\varepsilon_{0}=\left(\Delta e_{0} / V_{\text {тот }}\right) V_{0}$ where $V_{0}$ is the fully expanded nucleus volume $\left(V_{0} \gg V_{\mathrm{c}}\right)$ and $R$ the fraction of the large number of starting nuclei that achieves expansion per unit time, i. e. the activation rate.

In a simple picture, the activation rate $R$ depends on the frequency of attempts at overcoming a particular barrier, $v_{\mathrm{N}}$, and on the other hand, on the chance of success of a given fluctuation, which is the probability $p$ of an equilibrium fluctuation in energy greater than the energy $\Delta G_{\mathrm{a}}$. At a given temperature and pressure, $p$ is equal to the Boltzmann term $p=\exp -\Delta G_{\mathrm{a}} / k T$, so that :

$$
R=v_{\mathrm{N}} \exp -\left(\Delta G_{\mathrm{a}} / k T\right) .
$$

Thermal energy is distributed among polymer bonds through the various modes of vibration allowed inside their cage well. Roughly speaking, these modes can be featured by a bond frequency typical of high temperature jumps, $v_{\mathbf{D}}$; it is similar to the atomic Debye frequency for a crystal and of the same order of magnitude $v_{\mathrm{D}} \simeq 10^{13} \mathrm{~s}^{-1}[13,16 \mathrm{c}]$ since the elastic constants are only a factor ten smaller and densities are quite comparable. Clearly $v_{D}$ is an upper bound to $v_{N}$ for it corresponds to uncorrelated segment motions. As shown below, a better estimate for $v_{\mathrm{N}}$ is to take as frequency of correlated vibrations within a cage of volume $V_{c}$, made of a small number $n$ of segments $(n<10)$, the cage ground frequency :

$$
\left.v_{\mathrm{N}} \simeq \frac{v_{\mathrm{D}}}{n}=v_{\mathrm{D}} \frac{\Omega}{V_{\mathrm{c}}} \quad \text { (typically } v_{\mathrm{N}} \simeq 0.1 v_{\mathrm{D}}\right)
$$

where $\Omega$ is the volume available to an individual segment in the polymer.

Suppose a ring made of $n$ bonds, of length $n a$, vibrates with some degree of correlation. The vibrational state has to be obviously the same every $n$ segments so that allowed wave lengths are proportional to $2 n a$; the lowest energy, longest wave length mode has $2 n a$ as wave length and $v_{\mathrm{N}}=S / 2 n a$ as frequency, with $S$ a sound velocity. By the same token, the Debye frequency is approximated by $v_{\mathrm{D}}=S / 2 a$. Hence $v_{\mathrm{N}}=v_{\mathrm{D}} / n$, a result already rationalized along similar lines by Friedel for the dislocation attempt frequency in crystals [19].

From the above arguments, an order of magnitude evaluation can be made for the waiting time $\tau_{\mathrm{def}} \simeq R^{-1}$ of the activation process, i. e. for over- coming a particular barrier. Let us consider for example a constant strain rate test. The fundamental relation :

$$
\dot{\varepsilon}=N \varepsilon_{0} v_{\mathrm{N}} \exp \left(-\frac{\Delta G_{\mathrm{a}}}{k T}\right)
$$

can now be written :

$$
\Delta G_{\mathrm{a}}=k T \ln \left(N \varepsilon_{0} v_{\mathrm{N}} / \dot{\varepsilon}\right)=\alpha k T
$$

where $\alpha$ is constant, like $\dot{\varepsilon}$. Because of the large value of $v_{\mathrm{N}}, \alpha$ is large and weakly dependent on $\dot{\varepsilon}$. The typical value $\alpha \simeq 20$ very currently satisfied by a number of materials [8b] appears to hold also for thermoplastics [7]. Therefore one can estimate :

$$
\tau_{\text {def }}=R^{-1}=v_{\mathrm{N}}^{-1} \exp \left(\frac{\Delta G_{\mathrm{a}}}{k T}\right)=v_{\mathrm{N}}^{-1} \exp \alpha
$$

with $v_{\mathrm{N}} \simeq 10^{12} \mathrm{~s}^{-1}, \alpha \simeq 20$, it comes $\tau_{\mathrm{def}} \simeq 10^{-3} \mathrm{~s}$.

5. Operational quantities and experimental procedures. - We would point out, in conclusion, the importance of checking carefully the self consistency of the measured activation parameters in order to be sure of their physical meaning, before speculating on their physical origin. This is necessary because different active mechanisms may be mixed up at the investigated temperature, with the result that experimental data relate then only to apparent parameters; these are a complex combination of elementary contributions, generally hard to find out. When only one molecular mechanism is acting, on the other hand, experimentally independent procedures should lead to the same consistent set of data. The activation free energy $\Delta G_{\mathrm{a}}(T)$ measured at yielding as a function of temperature provides generally a good test.

Operational parameters directly available by independent experiments are the activation volume $V_{\mathrm{a}}$ and enthalpy $\Delta H_{\mathrm{a}}$, from the stress and temperature dependence of strain-rate. Neglecting any possible $\sigma$ or $T$ dependence in the pre-exponential factor $N \varepsilon_{0} v_{\mathrm{N}}$ in equation (12), compared to the exponential behaviour, it is easy to see that :

$$
k T\left(\frac{\partial \ln \dot{\varepsilon}}{\partial \sigma_{\mathrm{a}}}\right)_{T}=V_{\mathrm{a}} ; \quad k T^{2}\left(\frac{\partial \ln \dot{\varepsilon}}{\partial T}\right)_{\sigma_{\mathrm{a}}}=\Delta H_{\mathrm{a}} .
$$

In this way, $V_{\mathrm{a}}$ and $\Delta H_{\mathrm{a}}$ can be obtained at yielding $\left({ }^{5}\right)$, at a given stress $\sigma_{\mathrm{a}}$ (the yield stress) and temperature. For example $V_{\mathrm{a}}$ is obtained during a constant strain-rate test either by strain-rate jumps, or by the stress relaxation method [20]. $\Delta H_{\mathrm{a}}$ is obtained similarly by temperature jumps, either from the ensuing rate change $\Delta \dot{\varepsilon}$, or from the yield stress $\left(^{5}\right)$ change

(5) An operational definition of yielding is to take the stress at which the activation volume levels down to a plateau versus strain [21]. This is physically meaningful and gives yield stress values at various temperatures, in comparable corresponding states. 
$\Delta \sigma_{\mathrm{a}}$. This latter method makes use of the general Maxwell relation which can be written for $\dot{\varepsilon}\left(\sigma_{\mathrm{a}}, T\right)$ at constant rate $\dot{\varepsilon}$ :

$$
\Delta H_{\mathrm{a}}=-V_{\mathrm{a}} T\left(\frac{\partial \sigma_{\mathrm{a}}}{\partial T}\right)_{\dot{\mathrm{\varepsilon}}} .
$$

This relation assumes no additional structure variable enters the strain-rate $\dot{\varepsilon}$; accordingly, $V_{\mathrm{a}}$ and $\left(\partial \sigma_{\mathrm{a}} / \partial T\right)$ should be taken at the same microstructural state, which means measured on the same sample, at a time and strain as close as possible (hence the convenience of the stress relaxation method).

Two independent ways of obtaining $\Delta G_{\mathrm{a}}$ are available, which should produce consistent results :

(a) either by measuring $V_{\mathrm{a}}$ and $\Delta H_{\mathrm{a}}$ at yielding, using equations (11), (14) and eventually (15) ;

(b) or by measuring $V_{\mathrm{a}}$ at yielding (Eq. 14) and integrating it over the whole yield stress range, from $\sigma_{\mathrm{a}}^{\mathrm{M}}\left(\right.$ at zero $\mathrm{K}$ ) to $\sigma_{\mathrm{a}}$ at $T$ (Eq. 8). However, because $\Delta G_{\mathrm{a}}$ is a function of both $\sigma_{\mathrm{a}}$ and $T$, some scaling by the shear modulus has first to be done. We show in appendix that the procedure used by Cagnon [11] for crystals can still hold approximately for thermoplastics. $\Delta G_{\mathrm{a}}$ can thus be obtained by graphical integration :

$$
\Delta G_{\mathrm{a}}=\frac{\mu}{\mu_{0}} \int_{S}^{S_{\mathrm{M}}} V_{\mathrm{a}}(S) \mathrm{d} S
$$

where $S=\sigma_{\mathrm{a}}\left(\mu_{0} / \mu\right), \mu_{0}$ and $S_{\mathrm{M}}$ referring to zero Kelvin. Practically $S_{\mathrm{M}}$ is not known, but $\Delta G_{\mathrm{a}}$ can be fitted to a given value obtained by $(a)$, for example at the highest stress measured. Comparisons between $(a)$ and $(b)$ values can then be made for all the other experimental points.

Finally a second way of testing the analysis is to check the plot of $\Delta G_{\mathrm{a}}$ versus $T$ is a straight line passing through the origin, in agreement with equation (13).

We would like to give here just a brief sketch of the methods for illustration of the above procedures,

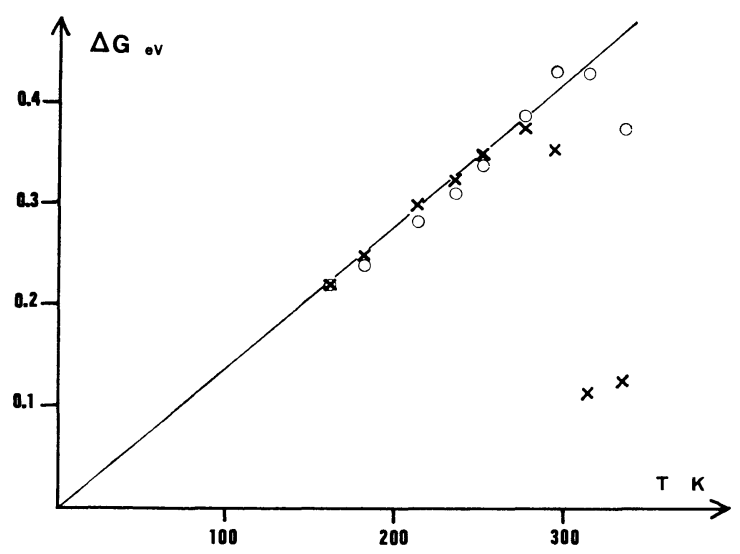

Fig. 1. - Activation free energy $\Delta G_{\mathrm{a}}$ measured at yielding as a function of temperature (compression at constant strain-rate) for atactic polystyrene [7] between $160 \mathrm{~K}$ and $335 \mathrm{~K}$. $\bigcirc \Delta G_{\mathrm{a}}$ values obtained by integration, equation (16). $\times \Delta G_{\mathrm{a}}$ values obtained by yield stress change after a temperature jump, equation (15). leaving for further publications [7] a more complete report on experimental data obtained for yielding in atactic polystyrene (P. S.) and polymethylmetacrylate (PMMA). These have been tested in uniaxial compression at a constant strain-rate, $\dot{\varepsilon}=10^{-4} \mathrm{~s}^{-1}$ and $\dot{\varepsilon}=3.6 \times 10^{-5} \mathrm{~s}^{-1}$ respectively. The activation free energy versus temperature curves are presented in figures 1 and 2 for these two thermoplastics.

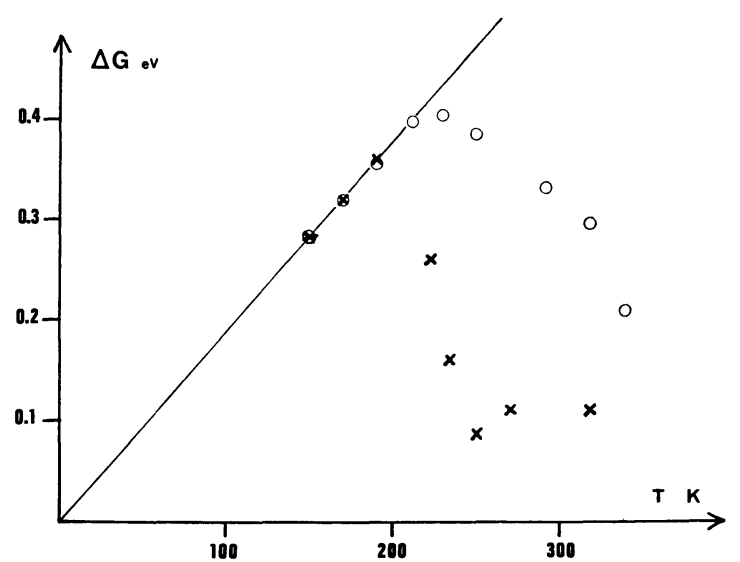

FIG. 2. - Same as figure 1 for atactic polymethylmethacrylate [7].

The figures show comparison between the temperature jumps (crosses, eq. (15)) and the integration method (circles, eq. (16)) in each case, after taking into account the temperature dependence of elastic modulus measured on similar samples at a frequency of $11 \mathrm{~Hz}$. The occurrence of fracture before yielding limited our investigations below $150 \mathrm{~K}$.

In the case of P. S., it is clearly seen that only one molecular mechanism is rate controlling up to $280 \mathrm{~K}$, with a good fit to equation (13) $\left(\Delta G_{\mathrm{a}}=\alpha k T, \alpha \simeq 17\right)$. Due to experimental constraints, in the case of PMMA, the linear temperature dependence of $\Delta G$ relies on only three low temperature data points, but with a straight line extrapolating correctly to the origin of coordinates and giving a reasonable slope $(\alpha \simeq 21)$ for equation (13). Accordingly, we think that in PMMA as well as in P. S., the strain rate is correctly described at low temperatures (below $280 \mathrm{~K}$ for P. S. and below $200 \mathrm{~K}$ for PMMA) by the Arrhenius law of equation (12), with a stress dependent activation energy, varying with $T$ as the elastic modulus does.

Beyond these temperatures, another mechanism seems to take place, with the result that the different ways of measuring $\Delta G_{\mathrm{a}}$ do not agree any more, nor does the linear relation-ship of $\Delta G_{\mathrm{a}}$ with $T$. While the temperature jump method still probably gives an apparent energy averaged between the two processes (the new one having a smaller energy), it may be that the integration method looses any meaning, for presumably the stress dependence is now only in the pre-exponential factor and any activation volume is without significance. 
Appendix. - Deriving THE ACTIVATION FREE ENERGY $\Delta G_{\mathrm{a}}\left(\sigma_{\mathrm{a}}, T\right)$ FROM INTEGRATING THE STRESS DEPENDENCE OF ACTIVATION VOLUME $V_{\mathrm{a}}$. - For crystals, Cagnon has shown [11] that scaling by the shear modulus produces a one variable function which is integrable :

$$
\Delta \Gamma=\frac{\mu_{0}}{\mu} \Delta G_{\mathrm{a}}, \quad S=\frac{\mu_{0}}{\mu} \sigma_{\mathrm{a}}
$$

with

$$
\left(\frac{\partial \Delta \Gamma}{\partial T}\right)_{S}=0 \text { and }\left(\frac{\partial \Delta \Gamma}{\partial S}\right)_{T}=-V_{\mathrm{a}}
$$

so that :

$$
\Delta \Gamma=\int_{S}^{S_{\mathrm{M}}} V_{\mathrm{a}}(S) \mathrm{d} S,
$$

hence :

$$
\Delta G_{\mathrm{a}}=\frac{\mu}{\mu_{0}} \int_{S}^{S_{\mathrm{M}}} V_{\mathrm{a}}(S) \mathrm{d} S .
$$

Here, the same procedure does not produce a one variable function, because of the rubber-like term; from equation (2), (3) and (6) :

$$
\Delta \Gamma=\int_{0}^{c}\left\{\frac{\mu_{0}}{\mu} h\left(\varepsilon_{\mathrm{s}}, T\right)-T \frac{\mu_{0}}{\mu} \frac{\partial S_{\mathrm{el}}}{\partial \varepsilon_{\mathrm{s}}}-S V_{\text {TOT }}\right\} \mathrm{d} \varepsilon_{\mathrm{s}}
$$

so that :

$$
\left(\frac{\partial \Delta \Gamma}{\partial S}\right)_{T}=-V_{\mathrm{a}}
$$

and

$$
\begin{aligned}
\left(\frac{\partial \Delta T}{\partial T}\right)_{S}=-\int_{0}^{c}\left[\frac{\mu_{0}}{\mu}-\right. & \left.\frac{T \mu_{0}}{\mu^{2}} \frac{\mathrm{d} \mu}{\mathrm{d} T}\right] \mathrm{d} S_{\mathrm{el}}= \\
& =-\mu_{0} \frac{\Delta S_{\mathrm{el}}}{\mu}\left(1-\frac{T}{\mu} \frac{\mathrm{d} \mu}{\mathrm{d} T}\right) .
\end{aligned}
$$

Therefore $\left(\frac{\partial \Delta \Gamma}{\partial T}\right)_{S}$ is proportional to

$$
-\Delta S_{\mathrm{el}} / \mu=\frac{k v_{\mathrm{e}} \Delta e_{0}}{2} \cdot \frac{V_{\mathrm{a}}}{\mu}
$$

according to equation (10). If the rubber-like term is small compared to the Hooke term, $-T\left(\partial S_{\mathrm{el}} / \partial \varepsilon_{\mathrm{s}}\right)<h$, it is reasonable to assume $V_{\mathrm{a}}=V_{\text {Tот }} \Delta \varepsilon_{\mathrm{s}}\left(\sigma_{\mathrm{a}}, T\right)$ to be proportional to the shear modulus $\mu$ (see Eq. (7)), as its only temperature dependent term. Therefore $V_{\mathrm{a}} / \mu$ and also $(\partial \Delta \Gamma / \partial T)$ has no temperature dependence.

On the other hand, at $\sigma_{\mathrm{a}}=\sigma_{\mathrm{a}}^{\mathbf{M}}, \sigma_{\mathrm{a}}^{\mathbf{M}}$ being the yield stress at $0 \mathrm{~K}$ and $T=0 \mathrm{~K}, \Delta G_{\mathrm{a}}$ is obviously zero, hence $\Delta \varepsilon_{\mathrm{s}}=0$ from equation (6), or $V_{\mathrm{a}}=0$ from equation (9). Since $V_{\mathrm{a}} / \mu$ has no temperature dependence, it follows that $\left(V_{\mathrm{a}} / \mu\right)=0$ for $\sigma_{\mathrm{a}}^{\mathrm{M}}$, or $S=S_{\mathrm{M}}$ whatever be $T$.

Let us now consider the three following states :

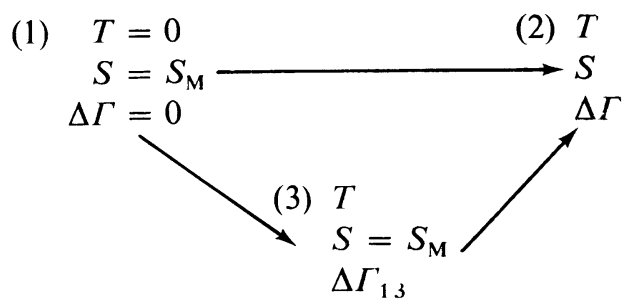

To compute $\Delta \Gamma$ at state (2), one has to integrate $\mathrm{d}(\Delta \Gamma)$ from state (1) to state (2). Since it is a state function, it is possible to do this integration via state (3). From (1) to (3) :

$$
\Delta \Gamma_{13}=\int_{0}^{T}\left(\frac{\partial \Delta \Gamma}{\partial T}\right)_{S=S_{M}} \mathrm{~d} T .
$$

But $\partial \Delta \Gamma / \partial T$ being proportional to $\left(V_{\mathrm{a}} / \mu\right)$ which is zero when $S=S_{\mathrm{M}}$, it itself zero, so $\Delta \Gamma_{13}=0$. From (3) to (2) :

$$
\Delta \Gamma_{32}=\int_{S_{M}}^{S}\left(\frac{\partial \Delta \Gamma}{\partial S}\right)_{T} \mathrm{~d} S .
$$

So that finally :

$$
\Delta \Gamma=\Delta \Gamma_{13}+\Delta \Gamma_{32}=\int_{S}^{S_{\mathrm{M}}} V_{\mathrm{a}}(S) \mathrm{d} S
$$

which is equivalent to equation (16). This result is thus established under the only assumption :

$$
-T \frac{\partial S_{\mathrm{el}}}{\partial \varepsilon_{\mathrm{s}}}=V_{\text {тот }} \frac{k T v_{\mathrm{e}} \Delta e_{0}}{2}<h\left(\varepsilon_{\mathrm{s}}, T\right) .
$$

But one has seen in part 3 that $\Delta G_{\mathrm{e} 1} \ll \Delta G_{\mu}$, which is, since the corresponding integrands are always positive, equivalent to :

$$
-T \frac{\partial S_{\mathrm{el}}}{\partial \varepsilon_{\mathrm{s}}} \ll h-\sigma_{\mathrm{a}} V_{\text {TOT }}<h .
$$

In conclusion, $\Delta G_{\mathrm{el}} \ll \Delta G_{\mu}$ is the condition for equation (16) to hold.

\section{References}

[1] Bowden, P. B., in The physics of Glassy Polymers, ed by. Haward, R. N. (Applied Science Publ. London), 1973, p. 279.

[2] Andrews, R. D. and Kazama, Y., J. Appl. Phys. 39 (1968) 4891.

[3] WARD, I. M., J. Mater. Sci. 6 (1971) 1397.
[4] Brady, T. E. and Yeh, G. S. Y., J. Appl. Phys. 42 (1971) 4622 .

[5] Evans, K. R., in Rate Processes in Plastic Deformation of Materials, ed. by Li J. C. M. and Mukherjee A. K. (Am. Soc. Metals, Metals Park, Ohio) 1975, p. 707.

[6] PINK, E., Mater. Sci. Eng. 24 (1976) 275. 
[7] Cavrot, J. P., Haussy, J., Lefebvre, J. M. and Escaig, B., to appear.

[8] Kocks, U. F., Argon, A. S., and Ashry, M. F., Thermodynamics and Kinetics of slip in Prog. in Mater. Sci. 19 (1975) ; (b) p. 242.

[9] Schoеcк, G., Phys. Status Solidi 8 (1965) 499.

[10] Surek, T., Luton, M. J. and Jonas, J. J., Philos. Mag. 27 (1973) 425.

[11] Cagnon, M., Philos. Mag. 24 (1971) 1465.

[12] Li, J. C. M., Pampillo, C. A. and Davis, L. A., in Deformation and Fracture of High Polymers, ed. by HenningKausch, H., Hassell, J. A., Jaffee, R. I. (Plenum Press) 1973, p. 239.

[13] Argon, A. S., Philos. Mag. 28 (1973) 839.
[14] Bowden, P. B. and Raha, S., Philos. Mag. 29 (1974) 149.

[15] Thierry, A., Oxborough, R. J. and Bowden, P. B., Philos. Mag. 30 (1974) 527.

[16] Bueche, F., Physical Properties of Polymers (Interscience Publ.) 1962, p. 108 ; (b) p. 247 ; (c) p. 89.

[17] LI, J. C. M., J. Appl. Phys. 42 (1971) 4543.

[18] Treloar, L. R. G., in The Physics of Rubber Elasticity (Clarendon Press, Oxford) 1975, p. 84.

[19] Friedel, J., in Dislocations (Addison Wesley Publ.) 1964, p. 66.

[20] Guiv, F. and Pratt, P. L., Phys. Status Solidi 6 (1964) 111.

[21] Farvacque, J. L., Crampon, J., Doukhan, J. C. and EscaIG, B., Phys. Status Solidi (a) 14 (1972) 623. 